\title{
Patient activation and its association with symptom burden and quality of life across the spectrum of chronic kidney disease stages in England
}

Winnie Magadi ${ }^{1 *}$, Courtney J. Lightfoot ${ }^{2}$, Katherine E. Memory², Shalini Santhakumaran', Sabine N. van der Veer ${ }^{3}$, Nicola Thomas ${ }^{4}$, Rachel Gair ${ }^{1}$ and Alice C. Smith ${ }^{2}$

\begin{abstract}
Background: The knowledge, skills, and confidence to manage one's own health is termed patient activation and can be assessed using the Patient Activation Measure (PAM). This measure is increasingly recommended for use in chronic kidney disease (CKD), but there is a need to better understand patient activation within this population. This work aimed to explore the association of PAM with patient-reported outcomes, namely symptom burden and healthrelated quality of life (HRQoL), to understand the relationship between patient activation and outcomes which are of importance to people with CKD.
\end{abstract}

Methods: Non-dialysis, dialysis, and kidney transplant patients from 14 renal units across England completed a survey comprising questionnaires assessing patient activation, symptom burden, and HRQoL.

Latent class analysis (LCA) was used to determine HRQoL and symptom burden subgroups in the data. Multinomial logistic regression analyses were performed to investigate the associations between patient activation and symptom burden and HRQoL classes separately, adjusting for age, gender, ethnicity, deprivation and treatment modality.

Results: Three thousand thirteen participants (mean age 61.5 years, $61.8 \%$ males, and $47 \%$ haemodialysis) were included in the analysis. Patient activation was strongly associated with both the HRQoL and symptom burden classes identified, with highly activated patients more likely to report higher HRQoL ( $P=<0.0001$; OR 29.2, 95\% Cl 19.5-43.9) and fewer symptoms ( $P=<0.0001$; OR 25.9, 95\% Cl 16.8-40.2).

Conclusion: Lower activation levels are associated with a higher symptom burden and reduced HRQoL across the trajectory of CKD stages and treatment modalities. Therefore, targeted and holistic self-management support focussing on improving activation may have the potential to improve aspects of health experience which are valued by individuals living with kidney disease.

Keywords: Patient activation, Symptom burden, Quality of life, Chronic kidney disease, Patient reported outcomes

*Correspondence: Winnie.Magadi@renalregistry.nhs.uk

1 UK Renal Registry, Brandon House Building 20A1, Southmead Road, Bristol BS34 7RR, UK

Full list of author information is available at the end of the article

\begin{abstract}
Introduction
The ability to manage one's own health is a key determinant in improving long-term health outcomes and quality of life (QoL) for a variety of chronic health conditions [1]. The concept of patient activation describes the knowledge, skills and confidence to manage one's own health
\end{abstract}


and healthcare [2, 3]. The most widely used tool for assessment of activation is the Patient Activation Measure (PAM) [2]. The PAM categorises individuals into one of four activation levels ranging from Level 1 (passive and lacking knowledge and skills) to Level 4 (active, wellinformed and competent). Higher levels of activation are often associated with lower healthcare costs [4] and improved health outcomes $[5,6]$. Individuals described as being highly activated are also more likely to participate in healthy lifestyle behaviours [7] and access health services including check-ups, screening and immunisations $[3,8]$.

Patient activation is increasingly acknowledged to underpin self-management [9]. In order to effectively manage long-term conditions such as chronic kidney disease (CKD), individuals are required to take an active role in their health using skills developed through information and support obtained from various educational and healthcare resources [10]. In CKD, poor engagement with self-management behaviours are associated with poor clinical outcomes such as progression to end-stage kidney disease (ESKD), cardiovascular disease, and death [11]. Patient activation has been used to tailor self-management support interventions to improve behavioural and health-related outcomes for patients with CKD [12]. The aim of increasing activation levels has also been incorporated into policies involving CKD populations [13].

With the application of the PAM in CKD being increasingly recommended [9], and recent validation in CKD [14], there is a need to better understand the factors which influence or are influenced by patient activation within this population. While clinical and cost outcomes are important to clinicians, healthcare provider organisations and policy makers, they are not necessarily rated as the most valued considerations for those living with the disease. There is now increasing global recognition of the need to incorporate the patient perspective in research and care planning and delivery in order to achieve improvements that provide genuine benefit in aspects of health and life which matter to the individual [15]. Patient-reported outcomes (PROs) such as health-related QoL (HRQoL) and symptom burden are such priorities identified by kidney patients themselves [16]. The need for a more personalised and patient-centred approach to health and care has been recognised in the NHS longterm plan with the introduction of a Comprehensive Model for Personalised Care [17]; this model of care is intended to support self-management, improve health and wellbeing outcomes, and quality of care, particularly for those with long-term conditions such as CKD [17]. Given the increasing prioritisation of patient activation and PROs, such as HRQoL and symptom burden, there is a need to understand the relationship between these factors to improve patient care and develop future healthpromotion and self-management interventions.

The Transforming Participation in CKD (TP-CKD) programme gathered Patient Reported Outcome Measure (PROM) data from an English population, comprising people with $C K D$, including those not requiring dialysis, receiving dialysis and kidney transplant recipients [18]. The current study aimed to explore activation levels and associated factors including PROs in the TP-CKD cohort. It was hypothesised that more activated individuals living with CKD would have a higher HRQoL and lower symptom burden than less activated individuals.

\section{Methods}

\section{Study design and setting}

This study utilised secondary data from the UK Renal Registry (UKRR) (ref: UKRR ILD24) collected as part of the national TP-CKD service evaluation programme. The programme, involving patients, carers, clinicians and commissioners, was based on a multi-centre, longitudinal cohort of CKD patients, either on renal replacement therapy (RRT; dialysis or transplantation) or not on RRT (termed non-dialysis), managed in secondary care. Participants were recruited from 14 renal units across England and completed a paper survey distributed by local renal teams between December 2015 and December 2017 [18]. Each survey comprised three questionnaires assessing patient activation, symptom burden and HRQoL. Once completed, the surveys were scanned by the UKRR into electronic format to allow linkage to other UKRR data items used in the study.

\section{Participants}

Inclusion criteria included: (1) patients with any stage of CKD or receiving any form of RRT in renal clinics in secondary care; (2) $\geq 18$ years; and 3 ) implicitly consented to their patient-reported outcome data being held by the UKRR.

\section{Demographics}

Demographics (date of birth, gender, ethnicity and index of multiple deprivation area), as well as treatment modality, were obtained by linking the participant's survey data to UKRR data using their unique NHS number. The UKRR only has full coverage of people on RRT, thus, individuals lacking information about RRT modality were assigned to the "non-dialysis" cohort, although no data on their stage of CKD was available.

\section{Patient activation measure (PAM)}

The 13-item PAM version, which draws on concepts such as health locus of control and self-efficacy in managing 
health behaviours, was used to assess patient activation [19]. Responses for each item were given on a 4-point Likert scale (( 1 strongly disagree to 4 strongly agree, with an additional "not applicable" option), assigning scores from 1 to 4 respectively. Raw scores were then converted to a scale of $0-100$ and an activation level of 1 to 4 indicating low to high activation. The activation groups are described as follows: Level 1 (score $\leq 47)$ consists of patients who do not believe they have an important role in their health; Level 2 (score 47.1-55.1) describes patients lacking in confidence or knowledge to take action; Level 3 (score 55.2-67.0) comprises patients starting to take action while Level 4 (score $\geq 67.1$ ) includes patients who maintain active behaviour. The PAM has recently been validated in the CKD population [14].

\section{EuroQol- 5 dimension (EQ-5D-5L)}

HRQoL was assessed using the EQ-5D-5L questionnaire, which measures 5 dimensions: mobility, self-care, usual activities, pain/discomfort, and anxiety/depression [20]. This is a widely used, validated measure of health status that can be standardised to different populations [21]. Participants rated each dimension on a scale from 1 (no problem) to 5 (unable). Responses to the five dimensions were combined into a 5-digit score to describe an individual's health state, with ' 11111 ' indicating 'no problems at all' and ' 55555 ' indicating 'extreme problems' [20]. A single utility score was then assigned to each combination using a scoring algorithm and based on the UK value set.

\section{Palliative care outcome scale-symptom (POS-S) renal}

Symptom burden was assessed using the 17-symptom POS-S Renal questionnaire [22]. Respondents indicated the extent to which they were bothered by each symptom over the last week on a scale from 0 (not at all) to 4 (overwhelmingly).

\section{Statistical analysis}

Descriptive analyses were performed for demographic parameters to compare characteristics of individuals in each of the four patient activation groups. Inferential statistics were also performed for outcome variables, including calculating mean scores for all dimensions of EQ-5D-5L and all POS-S Renal items and comparing them among the groups.

Latent class analysis (LCA) was utilised to determine whether HRQoL and symptom burden subgroups existed in the data, and if so, identify classes that best described the data. Latent class analysis is a statistical modelling approach which aims to find heterogeneity within the population by classifying individuals into unobserved groupings (latent classes) based on similar patterns of observed cross-sectional and/or longitudinal data [23]. As such, the goal is to probabilistically assign individuals into subpopulations by inferring each individual's membership to latent classes from the data. Thus, we conceptualized HRQoL and symptom burden as forming distinct categories or typologies as the use of raw scores may mask important differences among patients i.e. classes who self-report different types of limitations. We ran a 2, 3 and 4-class model to determine how many classes best described the subgroups in the population. The Bayesian Information Criteria (BIC) was used to determine goodness of fit [24]. These analyses were conducted using a SAS procedure developed by The Methodology Centre, PROC LCA [25].

Multinomial regression models were then developed to investigate: 1 ) the associations between patient activation and HRQoL classes and 2) the associations between patient activation and symptom burden classes. To handle missing data, we used multiple imputation with fully conditional specification, assuming the data were missing at random. We carried out 20 imputations using all available data on activation levels, symptom scores, HRQoL scores, and clinical and demographic data as predictor variables. Participants who had missing data on all the HRQoL and/or all the symptom burden items were excluded from the imputation and analyses. We checked that the distributions and correlations between variables were consistent between imputed and observed data. For both models, we controlled for the following factors; age, gender, ethnicity, treatment modality and index of multiple deprivation area quintile [26] (proxy of socioeconomic status derived from postcode, with higher quintiles representing more social deprivation). The PROC MIANALYZE procedure in SAS was then utilised to obtain the pooled parameter estimates and the variance information from the 20 imputations we ran prior [27]. We used SAS version 9.4 for all analyses.

\section{Results}

\section{Patient characteristics}

A total of 312 participants were excluded due to refused consent $(n=1)$, aged $<18(n=8)$, missing all symptom burden questions $(n=243)$ and missing all EQ-5D-5L questions $(n=60)$. Thus, the final study sample comprised 3013 participants who had similar demographic characteristics to the overall CKD and RRT population in the UK in 2016 (see Additional File 1 Table s2). Participant characteristics are displayed in Table 1. In summary, the mean age was 61.5 years, $61.8 \%$ were male, $81 \%$ white and $47 \%$ on haemodialysis. A third (34\%) of participants were categorised into PAM Level 3 (i.e. taking action to manage their own health). Mean scores for HRQoL and symptom burden dimensions were generally highest in 
Table 1 Baseline characteristics, presented with column percentages unless indicated otherwise

\begin{tabular}{|c|c|c|c|c|c|c|}
\hline${ }^{\mathrm{a}}$ Total N & $\begin{array}{l}\text { All } \\
3013(100)\end{array}$ & $\begin{array}{l}\text { PAM Level } 1 \\
756(25.1)\end{array}$ & $\begin{array}{l}\text { PAM Level } 2 \\
571(19.0)\end{array}$ & $\begin{array}{l}\text { PAM Level } 3 \\
1023(34.0)\end{array}$ & $\begin{array}{l}\text { PAM Level } 4 \\
526(17.5)\end{array}$ & $\begin{array}{l}\text { Missing } \\
137(4.5)\end{array}$ \\
\hline \multicolumn{7}{|l|}{ Covariates } \\
\hline \multicolumn{7}{|l|}{ Treatment type } \\
\hline Haemodialysis & 1415 (47.0) & $508(67.2)$ & $284(49.7)$ & $417(40.8)$ & $149(28.3)$ & $57(41.6)$ \\
\hline Peritoneal dialysis & $122(4.0)$ & $22(2.9)$ & $25(4.4)$ & $46(4.5)$ & $27(5.1)$ & $2(1.5)$ \\
\hline Transplant & $816(27.1)$ & $90(11.9)$ & $120(21.0)$ & $321(31.4)$ & $248(47.1)$ & $37(27.0)$ \\
\hline Non-dialysis & $660(21.9)$ & $136(18.0)$ & $142(24.9)$ & $239(23.4)$ & $102(19.4)$ & $41(29.9)$ \\
\hline \multicolumn{7}{|l|}{ Age (years) } \\
\hline 18-44 & $496(16.5)$ & $93(12.3)$ & $78(13.7)$ & $176(17.2)$ & $132(25.1)$ & $17(12.4)$ \\
\hline $45-54$ & $524(17.4)$ & $100(13.2)$ & $86(15.1)$ & $198(19.4)$ & $124(23.6)$ & $16(11.7)$ \\
\hline $55-64$ & $650(21.6)$ & $165(21.8)$ & $125(21.9)$ & $212(20.7)$ & $115(21.9)$ & $33(24.1)$ \\
\hline $65-74$ & $663(22.0)$ & $182(24.1)$ & $139(24.3)$ & $225(22.0)$ & $88(16.7)$ & $29(21.2)$ \\
\hline $75+$ & $678(22.5)$ & $216(28.6)$ & $143(25.0)$ & $211(20.6)$ & $67(12.7)$ & $41(29.9)$ \\
\hline Missing & $2(0.1)$ & $0(0)$ & $0(0)$ & $1(0.1)$ & $0(0)$ & $1(0.7)$ \\
\hline \multicolumn{7}{|l|}{ Gender } \\
\hline Male & $1568(52.0)$ & $411(54.4)$ & $292(51.1)$ & $531(51.9)$ & $270(51.3)$ & $64(46.7)$ \\
\hline Female & 970 (32.2) & $237(31.3)$ & $186(32.6)$ & $327(32.0)$ & $179(34.0)$ & 41 (29.9) \\
\hline Missing & $475(15.8)$ & $108(14.3)$ & $93(16.3)$ & $165(16.1)$ & 77 (14.6) & $32(23.4)$ \\
\hline \multicolumn{7}{|l|}{ Ethnicity } \\
\hline White & 2036 (67.6) & 468 (61.9) & $381(66.7)$ & 718 (70.2) & $395(75.1)$ & $74(54.0)$ \\
\hline Asian & $285(9.5)$ & $119(15.7)$ & $44(7.7)$ & $80(7.8)$ & $26(4.9)$ & $16(11.7)$ \\
\hline Black & $148(4.9)$ & $42(5.6)$ & $38(6.7)$ & $41(4.0)$ & $18(3.4)$ & $9(6.6)$ \\
\hline Chinese & $14(0.5)$ & $3(0.4)$ & $2(0.4)$ & $6(0.6)$ & $3(0.6)$ & $0(0)$ \\
\hline Other & $33(1.1)$ & $12(1.6)$ & $8(1.4)$ & $5(0.5)$ & $3(0.6)$ & $5(3.6)$ \\
\hline Missing & $497(16.5)$ & $112(14.8)$ & $98(17.2)$ & $173(16.9)$ & $81(15.4)$ & $33(24.1)$ \\
\hline \multicolumn{7}{|l|}{ IMD } \\
\hline Quintile 1 (least deprived) & $462(15.3)$ & $85(11.2)$ & $86(15.1)$ & $172(16.8)$ & $108(20.5)$ & $11(8.0)$ \\
\hline Quintile 2 & $506(16.8)$ & $104(13.8)$ & $93(16.3)$ & $177(17.3)$ & $113(21.5)$ & $19(13.9)$ \\
\hline Quintile 3 & $495(16.4)$ & $117(15.5)$ & $100(17.5)$ & $163(15.9)$ & $95(18.1)$ & $20(14.6)$ \\
\hline Quintile 4 & $625(20.7)$ & $170(22.5)$ & $103(18.0)$ & $221(21.6)$ & $103(19.6)$ & $28(20.4)$ \\
\hline Quintile 5 (most deprived) & $886(29.4)$ & $272(36.0)$ & $184(32.2)$ & $277(27.1)$ & $98(18.6)$ & $55(40.1)$ \\
\hline Missing & $39(1.3)$ & $8(1.1)$ & $5(0.9)$ & $13(1.3)$ & $9(1.7)$ & $4(2.9)$ \\
\hline
\end{tabular}

${ }^{a}$ The percentages reported for the total number of participants in each PAM level are the row percentages

the least activated group (PAM Level 1) and lowest in the most activated group (PAM Level 4) as shown in Table 2.

\section{Patient activation}

Those receiving haemodialysis comprised a much larger proportion of individuals in PAM Level 1 (67.2\%) compared to those in PAM Level 4 (28.4\%). In contrast, transplant patients represented $11.9 \%$ of those in PAM Level 1 and $47.1 \%$ in PAM Level 4. For patients not on RRT, the proportion in each activation group was fairly similar.

In terms of age, the distribution in the highest and lowest activation groups differed, with those in the youngest age category (18-44years) making up around a quarter of individuals in PAM Level 4 and only about half of that (12.3\%) in PAM Level 1. Conversely, for the oldest age category $(75+)$, a higher proportion was found in PAM Level 1 (28.6\%) compared to PAM Level 4 (12.7\%). For ethnicity, Asians appeared to be less activated than Whites, representing 15.7\% of those in PAM Level 1 and only $4.9 \%$ in PAM level 4 . In contrast, the proportion of white individuals was higher in PAM Level 4 (75.1\%) than in PAM Level 1 (61.9\%). Lastly, individuals in the most deprived quintile appear to be less activated, making up $36 \%$ of those in PAM Level 1 and only half that in PAM level 4 (18.6\%).

Regarding HRQoL, mean scores for mobility in the EQ5D-5L questionnaire ranged from 3.2 in PAM Level 1 to 1.7 in PAM Level 4, indicating that the latter self-reported 
Table 2 Mean scores for health-related quality of life (EQ-5D-5L) dimensions and symptom burden (POS-S Renal) by patient activation level

\begin{tabular}{|c|c|c|c|c|c|c|}
\hline & All & PAM Level 1 & PAM Level 2 & PAM Level 3 & PAM Level 4 & Missing \\
\hline Total N & 3013 & 756 & 571 & 1023 & 526 & 137 \\
\hline \multicolumn{7}{|l|}{ Outcomes } \\
\hline EQ-5D-5L & \multicolumn{6}{|c|}{ Mean (SD) score ${ }^{\text {a) }}$} \\
\hline All dimensions & $2.1(0.9)$ & $2.8(0.9)$ & $2.2(0.8)$ & $1.9(0.8)$ & $1.5(0.7)$ & $2.5(1.1)$ \\
\hline Mobility & $2.4(1.2)$ & $3.2(1.1)$ & $2.6(1.1)$ & $2.2(1.1)$ & $1.7(1)$ & $2.8(1.4)$ \\
\hline Self-care & $1.7(1.0)$ & $2.4(1.2)$ & $1.6(0.9)$ & $1.4(0.8)$ & $1.2(0.6)$ & $2(1.3)$ \\
\hline Usual activities & $2.4(1.3)$ & $3.2(1.2)$ & $2.5(1.2)$ & $2.1(1.2)$ & $1.6(1.0)$ & $2.8(1.5)$ \\
\hline Pain/Discomfort & $2.2(1.1)$ & $2.8(1.2)$ & $2.3(1.1)$ & $1.9(1.0)$ & $1.7(1.0)$ & $2.4(1.3)$ \\
\hline Anxiety/Depression & $1.9(1.1)$ & $2.5(1.2)$ & $1.9(1.0)$ & $1.7(0.9)$ & $1.5(0.8)$ & $2.2(1.4)$ \\
\hline POS-S Renal & \multicolumn{6}{|c|}{ Mean (SD) score } \\
\hline All symptoms & $1.7(0.5)$ & $2.0(0.5)$ & $1.7(0.5)$ & $1.6(0.5)$ & $1.5(0.5)$ & $1.9(0.6)$ \\
\hline Pain & $1.1(1.1)$ & $1.6(1.2)$ & $1.1(1.1)$ & $1.0(1.1)$ & $0.7(1.0)$ & $1.2(1.2)$ \\
\hline Shortness of breath & $1.1(1.1)$ & $1.5(1.1)$ & $1.1(1.0)$ & $1.0(1.0)$ & $0.7(0.9)$ & $1.2(1.2)$ \\
\hline Weakness & $1.7(1.1)$ & $2.2(1.1)$ & $1.7(1.1)$ & $1.5(1.1)$ & $1.1(1.0)$ & $1.8(1.3)$ \\
\hline Nausea & $0.6(0.9)$ & $1.0(1.1)$ & $0.6(0.9)$ & $0.5(0.8)$ & $0.4(0.7)$ & $0.8(1.0)$ \\
\hline Vomiting & $0.3(0.7)$ & $0.5(0.9)$ & $0.3(0.7)$ & $0.2(0.6)$ & $0.2(0.6)$ & $0.5(0.8)$ \\
\hline Poor appetite & $0.8(1.1)$ & $1.2(1.2)$ & $0.8(1.0)$ & $0.7(1.0)$ & $0.4(0.8)$ & $1.0(1.2)$ \\
\hline Constipation & $0.6(1.0)$ & $1.0(1.2)$ & $0.6(0.9)$ & $0.5(0.9)$ & $0.4(0.8)$ & $0.8(1.2)$ \\
\hline Sore of dry mouth & $0.9(1.1)$ & $1.2(1.2)$ & $0.8(1.0)$ & $0.8(1.0)$ & $0.5(0.9)$ & $1.0(1.1)$ \\
\hline Drowsiness & $1.1(1.1)$ & $1.5(1.2)$ & $1.0(1.0)$ & $0.9(1.0)$ & $0.6(0.8)$ & $1.2(1.2)$ \\
\hline Poor mobility & $1.4(1.3)$ & $2.1(1.2)$ & $1.5(1.2)$ & $1.2(1.2)$ & $0.6(1.0)$ & $1.7(1.4)$ \\
\hline Itching & $1.0(1.2)$ & $1.4(1.3)$ & $1.0(1.1)$ & $0.9(1.1)$ & $0.7(1.0)$ & $1.1(1.2)$ \\
\hline Difficulty sleeping & $1.2(1.3)$ & $1.7(1.3)$ & $1.3(1.2)$ & $1.1(1.2)$ & $0.9(1.2)$ & $1.4(1.4)$ \\
\hline Restless legs & $1.0(1.2)$ & $1.4(1.3)$ & $0.9(1.1)$ & $0.8(1.1)$ & $0.6(1.0)$ & $1.1(1.3)$ \\
\hline Changes in skin & $0.7(1.0)$ & $1.0(1.1)$ & $0.7(1.0)$ & $0.7(1.0)$ & $0.5(0.9)$ & $0.9(1.2)$ \\
\hline Diarrhoea & $0.5(0.9)$ & $0.6(1.0)$ & $0.5(0.9)$ & $0.4(0.8)$ & $0.3(0.7)$ & $0.7(1.0)$ \\
\hline Feeling anxious & $1.0(1.2)$ & $1.6(1.3)$ & $1.0(1.0)$ & $0.8(1.0)$ & $0.5(0.9)$ & $1.2(1.4)$ \\
\hline Feeling depressed & $0.9(1.1)$ & $1.5(1.3)$ & $0.9(1.1)$ & $0.7(1.0)$ & $0.4(0.8)$ & $1.2(1.3)$ \\
\hline
\end{tabular}

Note: EQ-5D-5L EuroQOL Five Dimensions - 5 levels version, POS-S Renal Palliative care Outcome Scale-Symptom Renal, SD standard deviation

${ }^{a}$ The data presented are for participants who answered at least one item in the EQ-5D-5L and POS-S-Renal surveys. High scores indicate high symptom severity on the POS-S Renal and more problems with the items on the EQ-5D-5L

fewer problems in this area (Table 2). This pattern was consistent across all dimensions. We observed the same trend for the 17 items assessing symptom burden i.e. the more activated groups had lower scores indicating fewer symptoms experienced by the individual. The mean scores for pain, for example, ranged from 1.6 for PAM Level 1 compared to 0.7 for PAM Level 4.

\section{Health-related quality of life}

Results of the LCA established that a three-class solution provided a good fit for the HRQoL data as the BIC value reduced significantly from 2 to 3 classes, compared to 3 to 4 and 4 to 5 classes (BIC: 2 classes- 4017; 3 classes2987; 4 classes- 2747; 5 classes- 2723). Thus, when balancing fit and parsimony, we found that HRQoL is best separated into three distinct classes: 'poor', 'moderate', and 'good'. The analyses showed that the proportion of the cohort in each latent class for HRQoL were as follows; 'poor' (27.4\%), 'moderate' (35.5\%) and 'good' (37.1\%). For those in the 'good' class, over $95 \%$ of individuals reported experiencing no problems or slight problems across all dimensions of the EQ-5D-5L. Conversely, in the 'poor' class the majority of the patients reported moderate or severe problems (Fig. 1). This was more pronounced for mobility and usual activities with a larger proportion $(>70 \%)$ reporting severe or extreme problems compared to the other dimensions of HRQoL $(<50 \%)$.

\section{Symptom burden}

The LCA established a three-class solution for the symptom burden data. The proportion of patients classed as having 'few', 'some' and 'many' symptoms according to the LCA were $32.3,46.2$ and $21.5 \%$, respectively. The analyses showed that over $89 \%$ of those in the 'few' 


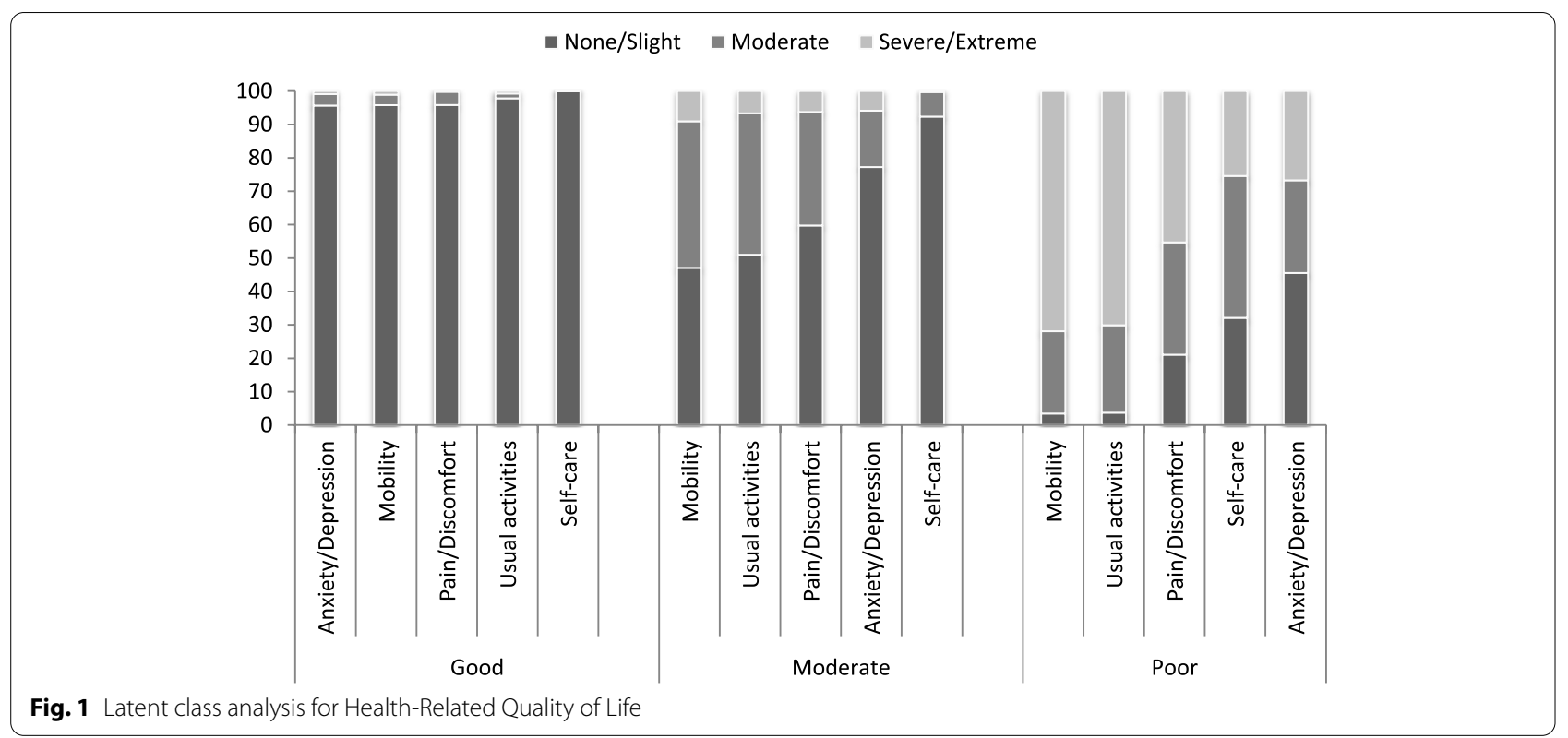

symptoms class experienced no symptoms or slight symptoms across all dimensions. In contrast, the proportion of those in the 'many' symptoms class with severe or extreme symptoms appeared to vary widely across the different components of this measure, ranging from $72.4 \%$ for weakness and $11.6 \%$ for vomiting (Fig. 2).

\section{Association between patient activation and HRQoL and symptom burden}

The multinomial regression analyses showed that patient activation was strongly associated with HRQoL.
Participants in PAM Level 4 have much greater odds of reporting 'good' HRQoL ( $\mathrm{P}=<0.0001$; OR $29.2,95 \% \mathrm{CI}$ 19.5-43.9) compared to those in PAM Level 1 (Table 3). A similar result was found for symptom burden with participants in PAM level 4 also having much greater odds of reporting few symptoms $(\mathrm{P}=<0.0001$; OR $25.9,95 \%$ CI 16.8-40.2) compared to those in PAM Level 1. Stratification of the analyses by treatment type confirmed the association between higher patient activation and better HRQoL and reduced symptom burden (see Additional File 1 Table s3). Thus, as observed with the whole

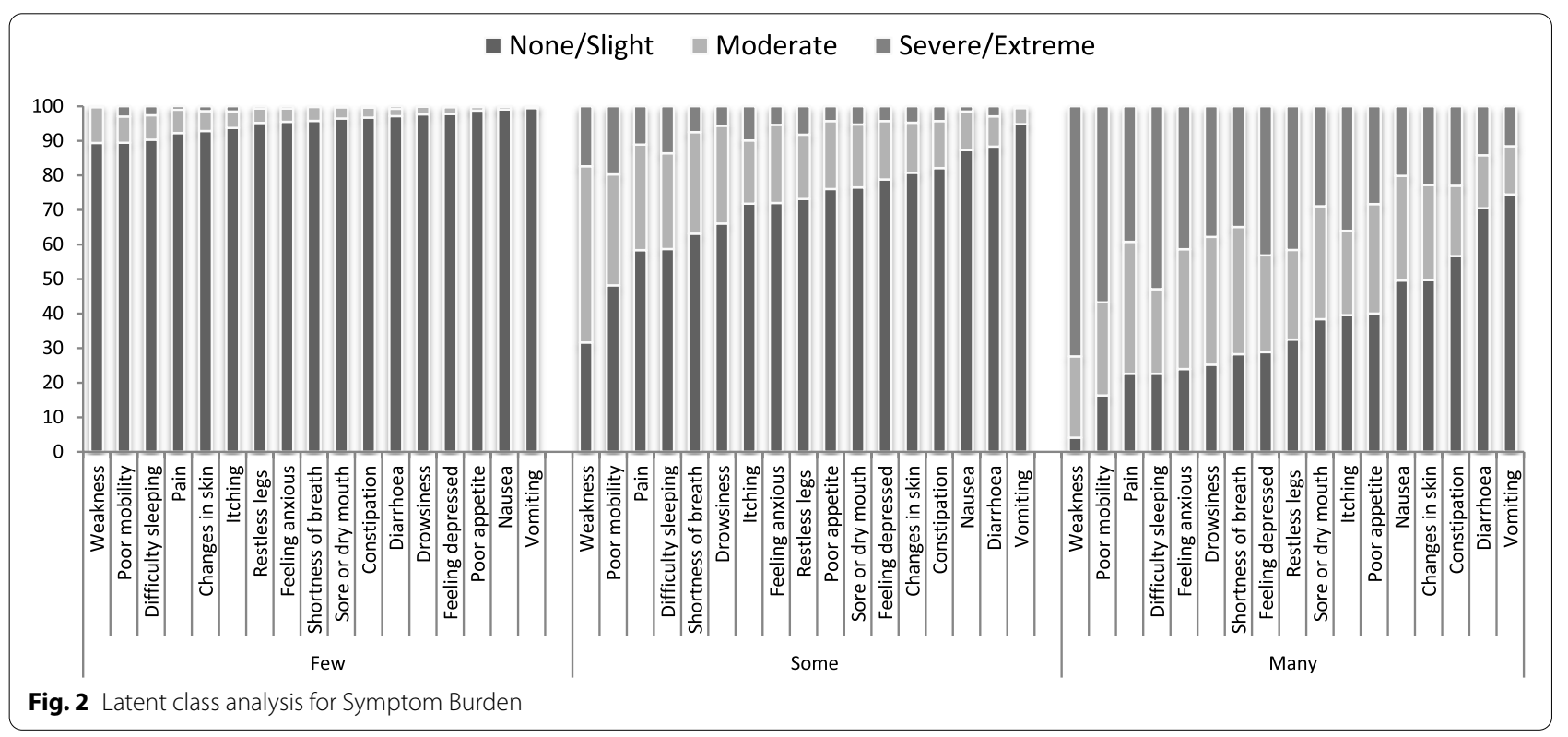


Table 3 Examining factors associated with patient activation; specifically health-related quality of life and symptom burden

\begin{tabular}{|c|c|c|c|c|}
\hline & \multicolumn{2}{|c|}{ Health-Related Quality of Life model } & \multicolumn{2}{|c|}{ Symptom burden model } \\
\hline & Good versus Poor & Moderate versus Poor & Few versus Many & Some versus Many \\
\hline & Odds ratio $(95 \% \mathrm{Cl})$ & Odds ratio $(95 \% \mathrm{Cl})$ & Odds ratio $(95 \% \mathrm{Cl})$ & Odds ratio $(95 \% \mathrm{Cl})$ \\
\hline \multicolumn{5}{|c|}{ Patient activation (ref: PAM Level 1) } \\
\hline PAM Level 2 & $4.9(3.5-6.8)$ & $2.8(2.2-3.6)$ & $5.1(3.6-7.3)$ & $2.8(2.2-3.8)$ \\
\hline PAM Level 3 & $11.6(8.5-15.7)$ & $3.3(2.6-4.2)$ & $9.1(6.6-12.5)$ & $3.0(2.4-3.9)$ \\
\hline PAM Level 4 & $29.2(19.5-43.9)$ & $3.9(2.7-5.8)$ & $25.9(16.8-40.2)$ & $5.0(3.4-7.5)$ \\
\hline Age $^{a}$ & $0.98(0.98-0.99)$ & $1.00(0.99-1.00)$ & $1.02(1.01-1.02)$ & $1.02(1.01-1.03)$ \\
\hline \multicolumn{5}{|l|}{ Gender (ref: Male) } \\
\hline Female & $0.6(0.5-0.7)$ & $0.8(0.7-1)$ & $0.5(0.4-0.7)$ & $0.7(0.6-0.9)$ \\
\hline \multicolumn{5}{|c|}{ Treatment type (ref: Haemodialysis) } \\
\hline Peritoneal dialysis & $1.6(0.9-2.8)$ & $1.4(0.9-2.4)$ & $0.6(0.4-1.2)$ & $1.0(0.6-1.6)$ \\
\hline Non-dialysis & $2(1.5-2.6)$ & $1.3(1-1.6)$ & $1.2(0.9-1.7)$ & $0.9(0.7-1.2)$ \\
\hline Transplant & $2.2(1.7-3)$ & $1.3(1-1.7)$ & $2.7(2-3.6)$ & $1.3(1-1.8)$ \\
\hline \multicolumn{5}{|c|}{ Deprivation ${ }^{\mathrm{b}}$ (ref: Quintile 1) } \\
\hline Quintile 2 & $0.7(0.5-1.1)$ & $1(0.7-1.5)$ & $0.9(0.6-1.3)$ & $0.8(0.5-1.2)$ \\
\hline Quintile 3 & $0.6(0.4-0.9)$ & $0.7(0.5-1)$ & $0.6(0.4-0.9)$ & $0.7(0.4-1)$ \\
\hline Quintile 4 & $0.4(0.3-0.6)$ & $0.7(0.5-1)$ & $0.6(0.4-0.9)$ & $0.8(0.5-1.1)$ \\
\hline Quintile 5 & $0.4(0.3-0.6)$ & $0.7(0.5-0.9)$ & $0.4(0.3-0.6)$ & $0.5(0.3-0.7)$ \\
\hline \multicolumn{5}{|l|}{ Ethnicity (ref: White) } \\
\hline Asian & $0.8(0.5-1.1)$ & $0.9(0.7-1.3)$ & $0.8(0.5-1.1)$ & $1.0(0.8-1.5)$ \\
\hline Black & $1.1(0.7-1.8)$ & $1.1(0.7-1.6)$ & $1.4(0.9-2.4)$ & $1.2(0.7-1.8)$ \\
\hline Chinese & $1.1(0.2-5.5)$ & $1.2(0.3-5)$ & $0.8(0.2-3.6)$ & $0.4(0.1-2)$ \\
\hline Other & $1.2(0.4-3.6)$ & $1.5(0.6-3.7)$ & $0.8(0.3-2.6)$ & $1.2(0.5-2.9)$ \\
\hline
\end{tabular}

a Values are odds ratios ( $95 \%$ confidence intervals), reflecting the odds of having a poor versus good and moderate health-related quality of life (HRQoL) and many versus few and some symptoms, per unit increase in age

${ }^{\mathrm{b}}$ Deprivation quintile 1 is the least deprived group and quintile 5 is the most deprived group

cohort, lower activation levels are associated with higher symptom burden and reduced HRQoL across treatment modalities. Due to small numbers in some of the categories in the stratified sample, the odds ratios for those in the peritoneal dialysis group are not presented in the table as these are unreliable.

\section{Discussion}

To our knowledge, this is the first study to explore the association between PRO factors (i.e. HRQoL and symptom burden) and patient activation across the CKD spectrum. Our findings demonstrate a strong link between patient activation and HRQoL, and symptom burden, with individuals who are highly activated reporting a better HRQoL and a lower symptom burden. This study also highlights certain factors such as being older, on haemodialysis, deprived, or from a non-white background which are associated with reduced levels of patient activation.

Low activation (PAM Level 1 or 2) was reported by almost half of CKD patients, indicating that they are passive recipients in their healthcare and lack the knowledge and confidence to take action [28]. Other CKD studies have reported similar activation levels, with 38 to $46 \%$ classed as PAM Level 1 and 2 [29-33], whilst others have reported $60 \%$ [34]. Our findings suggest that people with CKD have lower activation when compared to those with other chronic conditions, including inflammatory bowel disease, diabetes, HIV, and multiple sclerosis [35-38].

In our sample, haemodialysis patients were the least activated group. This is consistent with similar studies that have reported low activation levels in over half of haemodialysis patients [39]. Our finding of lower activation levels in the haemodialysis population compared to the non-dialysis or transplant groups mirrors that of other studies [29, 31, 32, 40]. The high morbidity [39] and heavy symptom and treatment burden [41] associated with dialysis may impact the ability to undertake selfmanagement tasks [42].

One of the key findings in our study was that the odds of having a better HRQoL and reduced symptom burden were much greater for patients who were more highly activated, after adjusting for age, gender, treatment modality, ethnicity, and deprivation. This is similar 
to previous studies which have shown an association between higher activation and a better QoL in older individuals with comorbidities [43], as well as patients with CKD [31, 34], multiple sclerosis [38] and inflammatory bowel disease [44].

To our knowledge, this is the first study to show an association between a reduced symptom burden and higher activation in CKD patients. An association between lower activation and worse self-reported health has previously been demonstrated in a CKD population [34] and a comorbid CKD and diabetic population [31]. This relationship may be driven by the fact that individuals who take an active role in their healthcare are more able to manage their symptoms and side effects. However, it is possible that individuals with a lower symptom burden feel more physically able to undertake self-management tasks [45]. Previous studies have demonstrated the impact of fatigue on haemodialysis patients' abilities to complete daily activities [46]. Indeed, the importance of providing patients with support to complete daily tasks is further emphasised through our findings that mobility and the ability to perform usual activities appear to be the key determinants of HRQoL. However, due to the overlapping components of HRQoL and symptom burden we were not able to carry out mediation analysis to explore the direction of the relationship between these variables.

\section{Strengths and limitations}

This study is strengthened by its large and nationwide sample of patients across different disease stages and treatment modalities, and as such it is the first study to present activation levels in a diverse kidney patient population including individuals on and off RRT. The utilisation of LCA allowed for assessment of heterogeneity in the HRQoL and symptom burden data and provided comprehensive evaluation of these measures in the study subjects based on all dimensions. Identifying subgroups in our data is important given that different groups of individuals in the population may require tailored support to increase patient activation levels.

As this was a secondary analysis of a national service evaluation programme, the recruitment strategy was not designed for research purposes and therefore no data is available for estimation of sample bias. Lack of Renal Registry information about patients who are not receiving RRT meant that it was not possible to know the CKD stage of the "non-dialysis" participants, and therefore no inference could be made about any association of CKD severity with the outcomes captured for this group. This study is also limited by our inability to adjust for comorbidity burden, despite it being well known that comorbidities have a great impact on HRQoL [47] as well on symptom burden [48]. This is primarily due to the reliability (or lack thereof) and completeness of the UKRR comorbidity data (mainly due to underreporting) to allow meaningful adjustment in this analysis. Another limitation is the cross-sectional study design which prevents the analysis of patient activation, HRQoL, and symptom burden over time.

In common with other studies involving self-reported data, we cannot exclude selection bias due to individuals with higher activation being more willing to participate and complete the outcome measures.

\section{Future work}

This overlooked topic merits more research and clinical attention to optimise resource targeting and deliver improved care quality and outcomes at lower costs. As patient activation underpins effective and sustained selfmanagement, its improvement provides an attractive goal for interventions aiming to promote and facilitate self-management behaviours. There is an urgent need to design, deliver and evaluate such interventions. Our observation of the association between higher activation levels and better patient-reported outcomes provides a potential engagement incentive for incorporation in such interventions, as patients often value these outcomes more than clinical benefits. Therefore, longitudinal studies should measure changes in activation over time to understand how patients transition from higher to lower activation, and how doing so impacts outcomes valued by the individual. Furthermore, future work should investigate whether symptom burden mediates the relationship between patient activation and QoL, including the possibility that improving patient reported outcomes could in itself lead to increased activation.

\section{Clinical relevance}

This population, particularly individuals receiving haemodialysis, display lower activation levels than other chronic disease populations, and are in need of targeted self-management support. The association of PAM levels with PROs suggests that approaches aiming to improve activation may have the potential to impact aspects of health and life which are valued by the individual as well as by clinicians and healthcare provider organisations. Patient education, resources, support, and advice with symptom management and physical rehabilitation may be required to support self-management behaviours and facilitate the transition to higher activation states in this population. Systematic development and evaluation of educational and behavioural support strategies focussing on individual needs and priorities are warranted, and 
could usefully incorporate the potential for improving valued PROs to encourage patient engagement.

\section{Summary/conclusion}

Patient activation was low in individuals with CKD across different treatment modalities, with nearly half of patients reporting low activation. Those who were highly activated had higher odds of better HRQoL and a lower symptom burden. Our findings warrant future studies into the impact of self-management support strategies on the patient experience as well as on clinical and cost outcomes.

\begin{abstract}
Abbreviations
Cl: Confidence interval; CKD: Chronic Kidney Disease; EQ-5D-5L: EuroQol 5 Dimension 5 Levels; HRQoL: Health-related Quality of Life; LCA: Latent Class Analysis; OR: Odds ratio; PAM: Patient Activation Measure; PRO: Patient Reported Outcome; POS-S Renal: Palliative care Outcome Scale - Symptoms Renal; RRT: Renal replacement therapy; TP-CKD: Transforming Participation in Chronic Kidney Disease; UKRR: United Kingdom Renal Registry.
\end{abstract}

\section{Supplementary Information}

The online version contains supplementary material available at https://doi. org/10.1186/s12882-022-02679-w.

Additional file 1 Table S1. Baseline characteristics for participants who had missing data on all EQ-5D-5L dimensions or POS-S Renal items who were excluded from the analyses, presented with column percentages unless indicated otherwise. Table S2. Comparison of baseline characteristics of all prevalent CKD and RRT patients in the UK Renal Registry at 31st December 2016 and the English TP-CKD study cohort (values are numbers (\% after excluding missing), unless indicated otherwise). Table S3. Multinomial regression analysis of the association between patient activation with health-related quality of life and symptom burden, stratified by treatment type.

\section{Acknowledgements}

We would like to thank all patients who completed the questionnaire and all renal unit staff who facilitated the data collection.

\section{Authors' contributions}

AS, SNvdV, NT and RG conceived the idea for the study. AS, WM and SNvdV were involved in designing the study. RG coordinated the data collection. WM and $\mathrm{SS}$ analysed the data, and WM, SS, CL, AS were involved in interpreting the results. WM, CL, KM and AS drafted the manuscript and all authors revised the manuscript critically for important intellectual content and approved the manuscript prior to submission.

\section{Funding}

$\mathrm{AS}$ and $\mathrm{CL}$ gratefully acknowledge research funding from the Stoneygate Trust and are supported by the Leicester NIHR Biomedical Research Centre. KM was supported by a Kidney Research UK Medical Student Bursary for 2019-2020 from the legacy of the late Professor Robin Eady. The views expressed in this publication are those of the authors and not necessarily those of the NHS, the NIHR Leicester BRC, the Stoneygate Trust or Kidney Research UK.

\section{Availability of data and materials}

The data underlying this article were provided by the UK Renal Registry by permission. Data will be shared on reasonable request to the corresponding author with permission of the UK Renal Registry.

\section{Declarations}

Ethics approval and consent to participate

The UKRR holds permissions under s251 of the NHS Act 2006, to gather, process, and share confidential patient information for the purposes of audit and research. These permissions are renewed annually by the Health Research Authority's Confidentiality Advisory Group. The collection and analysis of the data for research were carried out under the ethical permissions granted to the UKRR by the Research Ethics Committee. The completed questionnaires were submitted directly to the UKRR where they were linked with demographic data held by the UKRR. Once the linked dataset was created, it was analysed by a statistician employed by the UKRR. The aggregated results of these analyses were then sent to the research team for interpretation and inclusion in the final publication. Informed consent was received through implicit consent processes. All participants implicitly consented to their data being used for research purposes by the completing and returning the questionnaire. All methods were carried out in accordance with relevant guidelines and regulations.

\section{Consent for publication}

Not applicable.

\section{Competing interests}

The authors declare that they have no competing interests.

\section{Author details}

${ }^{1}$ UK Renal Registry, Brandon House Building 20A1, Southmead Road, Bristol BS34 7RR, UK. ' Leicester Kidney Lifestyle Team, Department of Health Sciences, University of Leicester, Leicester, UK. ${ }^{3}$ Centre for Health Informatics, Division of Informatics, Imaging and Data Sciences, Manchester Academic Health Science Centre, The University of Manchester, Manchester, UK. ${ }^{4}$ School of Health and Social Care, London South Bank University, London, UK.

Received: 8 March 2021 Accepted: 13 January 2022

Published online: 26 January 2022

\section{References}

1. Corben S, Rosen R. Self-management for long-term conditions. London: King's Fund; 2005.

2. Hibbard JH, Stockard J, Mahoney ER, Tusler M. Development of the patient activation measure (PAM): conceptualizing and measuring activation in patients and consumers. Health Serv Res. 2004;39(4 Pt 1):1005-26.

3. Hibbard JH, Greene J. What the evidence shows about patient activation: better health outcomes and care experiences; fewer data on costs. Health Aff (Millwood). 2013;32(2):207-14.

4. Greene J, Hibbard JH, Sacks R, Overton V, Parrotta CD. When patient activation levels change, health outcomes and costs change, too. Health Aff (Millwood). 2015;34(3):431-7.

5. Kinney RL, Lemon SC, Person SD, Pagoto SL, Saczynski JS. The association between patient activation and medication adherence, hospitalization, and emergency room utilization in patients with chronic illnesses: a systematic review. Patient Educ Couns. 2015;98(5):545-52.

6. Greene J, Hibbard JH. Why does patient activation matter? An examination of the relationships between patient activation and health-related outcomes. J Gen Intern Med. 2012;27(5):520-6.

7. Hibbard JH. Patient activation and the use of information to support informed health decisions. Patient Educ Couns. 2017;100(1):5-7.

8. Nijman J, Hendriks M, Brabers A, de Jong J, Rademakers J. Patient activation and health literacy as predictors of health information use in a general sample of Dutch health care consumers. J Health Commun. 2014;19(8):955-69.

9. Nair D, Cavanaugh KL. Measuring patient activation as part of kidney disease policy: are we there yet? J Am Soc Nephrol. 2020;31 (7):1435-43.

10. Bodenheimer T, Lorig K, Holman H, Grumbach K. Patient self-management of chronic disease in primary care. JAMA. 2002;288(19):2469-75.

11. Schrauben SJ, Hsu JY, Rosas SE, Jaar BG, Zhang X, Deo R, et al. CKD selfmanagement: phenotypes and associations with clinical outcomes. Am J Kidney Dis. 2018;72(3):360-70. 
12. Havas K, Douglas C, Bonner A. Meeting patients where they are: improving outcomes in early chronic kidney disease with tailored self-management support (the CKD-SMS study). BMC Nephrol. 2018;19(1):279.

13. National Quality Forum I. Patient reported outcomes (PROs) in performance measurement. Washington (DC): National Quality Forum; 2013. p. 2013.

14. Lightfoot CJ, Wilkinson TJ, Memory KE, Palmer J, Smith AC. Reliability and validity of the Patient Activation Measure in CKD. Under Review; 2021.

15. Tong A, Manns B, Wang AYM, Hemmelgarn B, Wheeler DC, Gill J, et al. Implementing core outcomes in kidney disease: report of the standardized outcomes in nephrology (SONG) implementation workshop. Kidney Int. 2018;94(6):1053-68.

16. Karet Frankl F, Coward R, Gallagher H, Hilton R, Loud F, Modi K, et al. UK Renal Research Strategy; 2016

17. England NHS. Universal personalised care: implementing the comprehensive model. London: NHS England; 2019.

18. Gair R, Stannard C, Wong E, Hawkins J, Van der Veer S, Karrington K, et al. Transforming participation in chronic kidney disease (TP-CKD): Programme report. In. Edited by Kidneys T; 2019.

19. Hibbard JH, Mahoney ER, Stockard J, Tusler M. Development and testing of a short form of the patient activation measure. Health Serv Res. 2005:40(6 Pt 1):1918-30.

20. Herdman M, Gudex C, Lloyd A, Janssen M, Kind P, Parkin D, et al. Development and preliminary testing of the new five-level version of EQ-5D (EQ-5D-5L). Qual Life Res. 2011;20(10):1727-36.

21. Szende A, Janssen B, Cabases J. Self-reported population health: an international perspective based on EQ-5D. Edn. Dordrecht (NL): Springer; 2014.

22. IPOS-Renal [https://pos-pal.org/maix/ipos-renal-in-english.php]

23. Berlin KS, Williams NA, Parra GR. An introduction to latent variable mixture modeling (part 1): overview and cross-sectional latent class and latent profile analyses. J Pediatr Psychol. 2013;39(2):174-87.

24. Schwarz G. Estimating the dimension of a model. Ann Stat. 1978;6(2):461-4.

25. Lanza ST, Dziak JJ, Huang L, Wagner A, Collins LM. Proc LCA \& Proc LTA users' guide (version 1.3. 2). University Park: The Methodology Center, Penn State; 2015

26. Ministry of Housing CLG: English indices of deprivation 2015. 2015.

27. SAS Institute Inc. SAS/STAT ${ }^{\circledR} 13.1$ user's guide. NC: SAS Institute Inc Cary; 2013

28. Hibbard JH, Mahoney ER, Stock R, Tusler M. Do increases in patient activation result in improved self-management behaviors? Health Serv Res. 2007;42(4):1443-63.

29. Johnson ML, Zimmerman L, Welch JL, Hertzog M, Pozehl B, Plumb T. Patient activation with knowledge, self-management and confidence in chronic kidney disease. J Renal Care. 2016;42(1):15-22.

30. Rivera E, Corte C, Steffen A, DeVon HA, Collins EG, McCabe PJ. Illness representation and self-care ability in older adults with chronic disease. Geriatrics (Basel). 2018;3(3):45.

31. Zimbudzi E, Lo C, Ranasinha S, Fulcher GR, Jan S, Kerr PG, et al. Factors associated with patient activation in an Australian population with comorbid diabetes and chronic kidney disease: a cross-sectional study. BMJ Open. 2017;7(10):e017695.

32. Hamilton AJ, Caskey FJ, Casula A, Inward CD, Ben-Shlomo Y. Associations with wellbeing and medication adherence in young adults receiving kidney replacement therapy. Clin J Am Soc Nephrol. 2018;13(11):1669-79.

33. Lo C, Zimbudzi E, Teede HJ, Kerr PG, Ranasinha S, Cass A, et al. Patientreported barriers and outcomes associated with poor glycaemic and blood pressure control in co-morbid diabetes and chronic kidney disease. J Diabetes Complicat. 2019;33(1):63-8.

34. Wilkinson TJ, Memory KE, Lightfoot CJ, Palmer J, Smith AC. Determinants of patient activation and its association with cardiovascular disease risk in chronic kidney disease: a cross-sectional study. Health Expect. 2021;39(4):1005-26.

35. Barnes EL, Long MD, Kappelman MD, Martin CF, Sandler RS. High patient activation is associated with remission in patients with inflammatory bowel disease. Inflamm Bowel Dis. 2019;25(7):1248-54.

36. Gleason KT, Tanner EK, Boyd CM, Saczynski JS, Szanton SL. Factors associated with patient activation in an older adult population with functional difficulties. Patient Educ Couns. 2016;99(8):1421-6.
37. Kendall CE, Shoemaker ES, Crowe L, MacPherson P, Becker ML, Levreault E, et al. Patient activation among people living with HIV: a cross-sectional comparative analysis with people living with diabetes mellitus. AIDS Care. 2018;30(11):1444-51.

38. Stepleman L, Rutter MC, Hibbard J, Johns L, Wright D, Hughes M. Validation of the patient activation measure in a multiple sclerosis clinic sample and implications for care. Disabil Rehabil. 2010;32(19):1558-67.

39. Van Bulck L, Claes K, Dierickx K, Hellemans A, Jamar S, Smets S, et al. Patient and treatment characteristics associated with patient activation in patients undergoing hemodialysis: a cross-sectional study. BMC Nephrol. 2018;19(1):126.

40. Magnezi R, Glasser S. Psychometric properties of the Hebrew translation of the patient activation measure (PAM-13). PLoS One. 2014;9(11):e113391.

41. Li H, Xie L, Yang J, Pang X. Symptom burden amongst patients suffering from end-stage renal disease and receiving dialysis: a literature review. Int J Nurs Sci. 2018;5(4):427-31.

42. Bristowe K, Horsley HL, Shepherd K, Brown H, Carey I, Matthews B, et al. Thinking ahead--the need for early advance care planning for people on haemodialysis: a qualitative interview study. Palliat Med. 2015;29(5):443-50.

43. Blakemore A, Hann M, Howells K, Panagioti M, Sidaway M, Reeves D, et al. Patient activation in older people with long-term conditions and multimorbidity: correlates and change in a cohort study in the United Kingdom. BMC Health Serv Res. 2016;16(1):582.

44. Munson GW, Wallston KA, Dittus RS, SperoffT, Roumie CL. Activation and perceived expectancies: correlations with health outcomes among veterans with inflammatory bowel disease. J Gen Intern Med. 2009;24(7):809-15.

45. Bringsvor HB, Langeland E, Oftedal BF, Skaug K, Assmus J, Bentsen SB. Effects of a COPD self-management support intervention: a randomized controlled trial. Int J Chron Obstruct Pulmon Dis. 2018;13:3677-88.

46. Horigan AE, Schneider SM, Docherty S, Barroso J. The experience and self-management of fatigue in patients on hemodialysis. Nephrol Nurs J. 2013:40(2):113-22 quiz 123.

47. Nelis SM, Wu Y-T, Matthews FE, Martyr A, Quinn C, Rippon I, et al. The impact of co-morbidity on the quality of life of people with dementia: findings from the IDEAL study. Age Ageing. 2019;48(3):361-7.

48. Wu M, Zan T, Zhao Q, Ge S, Li K, Li J. Symptom clusters and health-related quality of life in Chinese patients with chronic obstructive pulmonary disease. West J Nurs Res. 2020;42(6):437-45.

\section{Publisher's Note}

Springer Nature remains neutral with regard to jurisdictional claims in published maps and institutional affiliations.

Ready to submit your research? Choose BMC and benefit from

- fast, convenient online submission

- thorough peer review by experienced researchers in your field

- rapid publication on acceptance

- support for research data, including large and complex data types

- gold Open Access which fosters wider collaboration and increased citations

- maximum visibility for your research: over $100 \mathrm{M}$ website views per year

At BMC, research is always in progress.

Learn more biomedcentral.com/submissions 